\title{
Application of GA-based NMPC for pH Value Control in Sewage Disposal
}

\author{
Zhang Chao ${ }^{1}$, Zou Yingyong ${ }^{1}$ and $\mathrm{Pan} \mathrm{Su}^{2}$ \\ ${ }^{1}$ School of Electronic and Information Engineering, Chang Chun University, \\ Chang Chun, China \\ ${ }^{2}$ Jilin University, Jilin Province, P.R.China \\ 157075018@qq.com
}

\begin{abstract}
The process of $p H$ neutralization reaction in sewage disposal is characterized with highly nonlinearity and hysteretic nature. In the thesis, first of all, it is described for the process of neutralization reaction in sewage disposal as well as its mechanism model, analyzed them by putting forward a strategy of NMPC based on Method of Genetic Optimization. The resolution of uneasy nonlinear optimization is to be made by utilizing Genetic Algorithm for an overall optimization, simple controlled parameters and etc. Simulation experiment gives us an explanation for this method to very good track of the settings of $\mathrm{pH}$ value with fast response time, ensuring an effective control for $\mathrm{pH}$ value.
\end{abstract}

Keywords: sewage disposal; NMPC; GA; $p H$ value

\section{Introduction}

PH value control system plays an important part in an overall system of sewage disposal, but, due to highly nonlinearity as well as hysteretic nature in the process of neutralization reaction, enabling it an issue difficult to handle in modern controlling realm. In recent years, a traditional PID controlling system has been widely used, but very difficult in well-controlling those processes with highly non-linearity. Nonlinear Model Predictive Control (NMPC) is an effective way for resolution of a highly nonlinear controlling process, the key of which is to establish an applicable non-linearity controlling predictive model and the method of rational optimization. As a non-linearity controlling predictive model gives rise to non-convex optimization issue, also a traditional method difficult to get an overall optimal solution, recently, an optimal solution based on genetic algorithm finds its wide application on NMPC system. Genetic algorithm (GA) is an abstract concept of evolutionary process for an organism. It comprehensively simulates the natural selection and genetic mechanism to form a search algorithm with the features on "Generation\&Inspection". An overall optimal solution can be obtained by this genetic algorithm in such a larger probability. Population can be gradually evolved in a state equivalent to or close to an optimal value by such a series of genetic operations as selection, cross classification and mutation applied to the present population.

In accordance with the technical requirements stipulated in $\mathrm{pH}$ controlling system, combined NMPC with an advantage of genetic algorithm on optimization computation, genetic algorithm can be used to solve an issue for non-linear optimization, and then it can improve the control effect on $\mathrm{pH}$ value, to shorten the time of optimization solution.

\section{NMPC in the Process of Neutralization Reaction}

\section{1. pH Value Controlling Process in the Sewage Disposal}

Figure ure 1. is arranged for a certain sewage treatment plant. It adopts a block diagram 
of $\mathrm{pH}$ value control system in combination with PC and PLC in sewage disposal. This system consists of PC, PLC on site, data detection as well as actuating mechanism, in which PC conducts data-processing to be transmitted from PLC on site and then sends an operational directive to PLC. PLC receiving\&inspection equipment on site (e.g., Flow meter, $\mathrm{pH}$ meter and etc) is used for data measurement and control of actuating mechanism (e.g., metering pump and etc.,). Controlled variable in $\mathrm{pH}$ value control system means $\mathrm{pH}$ value in reaction tank that is regulated by the flux of neutralizing agent.

The key issue in the whole system is how the controlled variable can be calculated by collect data, due to highly nonlinearity and hysteretic nature in neutralization reaction process, so difficult to control it by regular algorithm (e.g., PID control) that NMPC is designed for the resolution of this issue.

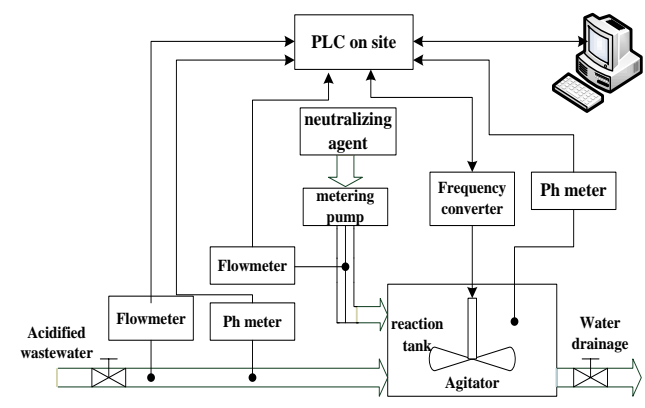

\section{Figure 1. pH Value Control System in Sewage Disposal}

\section{2. pH value Control System Model}

Actually, $\mathrm{pH}$ neutralization reaction in sewage disposal is a very complex one, in which CSTR is a typical system to be used for this process, and its structure is shown in the Figure 2.

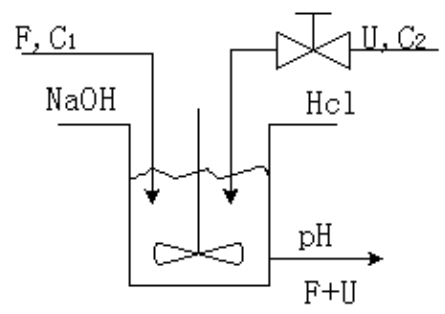

Figure 2. Structure Diagram of CSTR System

In the Figure 2., the flux of alkaline liquor ( $\mathrm{NaOH}$ ) is defined as $\mathrm{F}$ (constant quantity), and $C_{1}$ is a concentration of alkaline liquor; the flux of acid liquor $(\mathrm{Hcl})$ is defined as $\mathrm{F}$ (constant quantity), and $\mathrm{C}_{2}$ is a concentration of acid liquor; $\mathrm{pH}$ value in the eluate is defined as that of system output (i.e., controlled variable). This CSTR model can be described in equation 1.

$$
\left\{\begin{array}{l}
V(t) \frac{d \alpha(t)}{d t}=F C_{1}-(F+U(t)) \alpha(t) \\
V(t) \frac{d \beta(t)}{d t}=U(t) C_{2}-(F+U(t)) \beta(t)
\end{array}\right.
$$

Where: $V(t)$ is a volume of reaction tank at the moment $\mathrm{t} ; U(t)$ is a flux of acid liquor in the pipeline at the moment $\mathrm{t} ; \alpha(t)$ is an ion concentration sodium $\left(\mathrm{Na}^{+}\right)$at the moment $\mathrm{t} ; \beta(t)$ is an ion concentration sodium $\left(\mathrm{cl}^{-}\right)$at the moment $\mathrm{t}$.

It is assumed that $x=\beta-\alpha$, Equation1 can be obtained as follows: 


$$
V(t) \frac{d x(t)}{d t}=U(t) C_{2}-F C_{1}+(F+U(t)) x(t)
$$

It can be derived from the calculating equation for $\mathrm{pH}$ value as follows:

$$
x(t)=10^{y(t)-14}-10^{-y(t)}
$$

Where, $y(t)$ is $\mathrm{pH}$ value generated from the system at the moment $\mathrm{t}$. Equation 2 and Equation 3 are constituted as a mathematic model for description of $\mathrm{pH}$ value control system.

Equation 3 can be changed into the following conditions by discrediting between Equation 2 and Equation 3 together:

$$
\begin{gathered}
x(k+1) V(k+1)=2 x(k) V(k)+U(k) C_{2} T-F C_{1} T \\
y(k)=\frac{\log _{e}\left(5 \times 10^{13} \cdot x(k)-10^{7} \times \sqrt{\left.2.5 \times 10^{13} \cdot[x(k)]^{2}+1\right)}\right.}{\log _{e} 10}
\end{gathered}
$$

Equation 4 and Equation 5 will be constituted together to form a mathematic model for description of $\mathrm{pH}$ value control system. From the said mathematic model it can be seen that $\mathrm{pH}$ value $y(t)$ generated from the reaction tank is determined by state variable $x(t)$. When the flux of alkaline liquor $\mathrm{F}$ is fixed, $x(t)$ can be regulated by $U(t)$ in differential Equation 2, shown in Figure 3 for $\mathrm{pH}$ value characteristic curve. From Figure 3.-pH value characteristic curve it can be seen that the whole reaction process is characterized with highly non-linearity, in the approximate of neutral point obtained a higher sensitivity. Therefore, NMPC is the right choice.

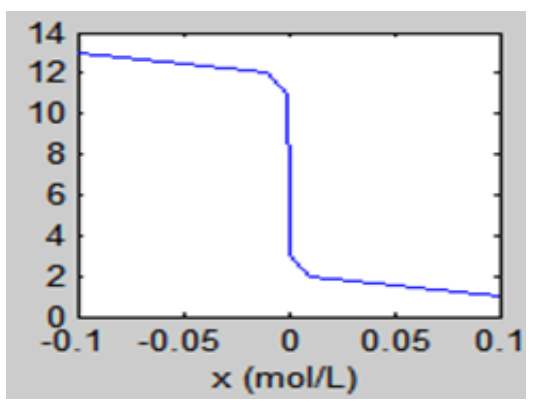

Figure 3. pH Value State Characteristics in the Reaction Tank

\subsection{NMPC in pH Neutralization Process}

According to said the system model for $\mathrm{pH}$ value control, a nonlinear model can be described for discrete time as follows:

$$
\begin{aligned}
& X_{k+1}=f\left(X_{k}, U_{k}, \delta_{k}\right) \\
& y_{k}=g\left(X_{k}\right)+\varepsilon_{k}
\end{aligned}
$$

Where, $X_{k}$ is a state variable at the moment $\mathrm{t}, U_{k}$ is an operating variable (i.e. input variable) at the moment $\mathrm{t}, y_{k}$ is controlled variable (i.e., $\mathrm{pH}$ value), $\delta_{k}$ and $\varepsilon_{k}$ are measurable noises at the moment $t$.

Provided that the difference between a predictive value at present output and measuring value is $b_{k}$, i.e.,

$$
b_{k}=y_{k}^{m}-y_{k}
$$

Then, after corrected the model, the predictive output for $j$ model will be obtained as 
follows:

$$
y_{k+j}=g\left(X_{k+j}\right)+b_{k}
$$

Quadric form objective function is the most widely used one that reflects the performance evaluation to better tracking expectations for output value. Defined objective function for NMPC can be obtained as follows:

$$
J=\sum_{j=1}^{P}\left\|e_{k+j}^{y}\right\| Q_{j}+\sum_{j=1}^{M-1}\left\|\Delta U_{k+j}\right\| S_{j}+\sum_{j=1}^{M-1}\left\|e_{k+j}^{U}\right\| R_{j}
$$

Where, $e_{k+j}^{y}=y_{k+j}-y_{s}$ is the difference between predictive output and output expectations, $e_{k+j}^{U}=U_{k+j}-U_{s}$ is the difference between predictive input and input expectations, $\Delta U_{k+j}$ is an increment of predictive input, $P$ is a predictive time domain, $M$ is a controlled time domain, $Q_{j}, s_{j}$ and $R_{j}$ are respectively defined as weighting coefficients at time varying, and $Q=\operatorname{diag}\left(Q_{1} Q_{2} \cdots Q_{P}\right)$, $S=\operatorname{diag}\left(S_{1} S_{2} \cdots S_{M-1}\right), R=\operatorname{diag}\left(R_{1} R_{2} \cdots R_{M-1}\right)$.

NMPC model in the process of $\mathrm{pH}$ neutralization reaction includes equality constraint and non-equality constraint conditions: equality constraints are defined in Equation 4 and Equation 5, and non-equality constraints can be obtained as follows:

$$
\begin{gathered}
\underline{y}_{j} \leq y_{k+j} \leq \bar{y}_{j} \quad(j=1,2, \cdots, P) \\
\underline{U} \leq U_{k+j} \leq \bar{U} \quad(j=0,1, \cdots, M-1) \\
\Delta \underline{U} \leq \Delta U_{k+j} \leq \Delta \bar{U} \quad(j=0,1, \cdots, M-1)
\end{gathered}
$$

According to the said objective function, equality constraint and non-equality constraint conditions in the NMPC model, optimal controlled variable $U_{k}$ can be obtained by optimization method. After input it into the calculating equation, output variable $y_{k+1}$ can be obtained, followed by the optimal controlled variable $U_{k+1}$ obtained from the moving optimization. In such case, sampling frequency can be repeatedly conducted to form an NMPC-based input sequence as follows. i.e.,:

$$
U^{M}=\left\{U_{k}, U_{k+1}, \cdots, U_{k+M-1}\right\}
$$

As nonlinear model and non-equality constraint condition are introduced into NMPC in the process of $\mathrm{pH}$ neutralization, enabling this solving process to be turned into a nonconvex solution process. General solving process is very likely to drop into a local optimal solution, rather than an overall optimal solution, and then influenced the controlled effects. For this reason, the method of genetic algorithm can be used in solving this NMPC model.

\section{Genetic Algorithm-based Optimization Solution}

Aiming at an issue difficult to conduct optimization solution by NMPC, the application of the method of genetic algorithm is to solve the said model, in which the solving procedure is defined as follows:

(1) At the sampling moment $K$, the difference between predictive output value and measuring value can be calculated at the moment $k-1$;

(2) Objective function in the NMPC can be defined as fitness function in the genetic algorithm, the minimum value in the Equation 10 obtained using genetic algorithm in conformity with the controlled sequence for constraints.

(1)The determination of initialization parameter used in the genetic algorithm: the size of population $M$, algebra of evolution termination T, chromosome length $L$, dimension of 
solution space $s(s=1)$, cross probability $P_{C}$, mutation rate $P_{m}$.

(2) Population Initialization: For those constraint condition applied in search algorithm with search space limitation, i.e., in the space of feasible solution, $M$ individual population can be randomly derived from $P_{i}(t), i=1, \cdots, M \quad t=1, \cdots, T \quad t=1, \cdots, T$. Output variable can be respectively Figure ured out after taken $P_{i}(t)$ as present controlled variable, the fitness value $f\left(P_{i}(t)\right)$ respectively calculated then:

(3) Selecting operations: the probability each individual selected is determined by Equation (15), from selecting operation generated a new population $P_{i}^{\prime}(t)$.

$$
P_{s}\left(P_{i}(t)\right)=\frac{f\left(P_{i}(t)\right)}{\sum_{i=1}^{M} f\left(P_{i}(t)\right)}, i=1,2, \cdots, M
$$

(4) Cross operations: new experimental individual $P_{i}{ }^{\prime \prime}(t)$ will be obtained from Equation(16)

$$
P_{i}^{\prime \prime}(t)= \begin{cases}P_{i}{ }^{\prime}(t), & x \leq P_{c} \\ P_{i}{ }^{\prime \prime}(t), & \text { otherwise }\end{cases}
$$

Where, $x$ is taken from $[0,1]$ in conformity with uniformly distributed random variable, while cross model is defined as uniformly distributed.

(5) Mutation operation: mutation can be obtained from Equation (17)

$$
P_{i j}{ }^{\prime \prime \prime}(t)=\left\{\begin{array}{ll}
1-P_{i j}{ }^{\prime \prime}(t), & x \leq P_{m} \\
P_{i j}{ }^{\prime \prime}(t), & \text { otherwise }
\end{array} \quad j=1, \cdots, L\right.
$$

Where, $j$ is defined as gene location of chromosome

(6) The said procedures (3) 5cannot be Repeatedly performed till obtained maximum evolution algebra or precision of objective function.

(3) Using $U_{k}$ value for controlled variable obtained from procedure (II) at the present moment evaluates the system output $y_{k+1}$ value in accordance with system control model, followed with next sampling cycle.

\section{Genetic Algorithm-based NMPC Structure}

NMPC system for control of $\mathrm{pH}$ value consists of reference tracking, moving optimization, predictive model, on-line calibration and $\mathrm{pH}$ neutralization model, in which its system structure is shown in Figure 4. Where, reference tracking is a smooth curve generated from the settings made in the system through present output values; an objective function in the moving optimization can be defined in Equation(10); genetic algorithm can be used for the solution in the moving optimization process.

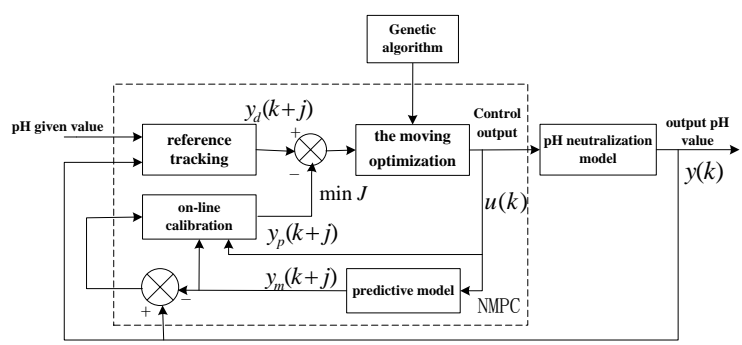

Figure 4. Genetic Algorithm-based NMPC Structure 


\section{Simulation Test}

In the system of simulation test, the initial condition can be given as below:

$V=1000 \mathrm{~L}, F_{1}=81 \mathrm{~L} / \mathrm{min}, C_{1}=0.3178 \mathrm{~mol} / \mathrm{L}, C_{2}=0.35 \mathrm{~mol} / \mathrm{L}$. The parameters are determined by genetic algorithm in time after time tests, among which the size of population: $M=50$; algebra of evolution termination: $T=100$, cross probability: $P_{c}=0.5$, mutation probability: $P_{m}=0.005$. The operating time for genetic algorithm: 3 seconds. Sampling cycle: 20 seconds, Figure 5 and Figure 6. Figure out the control effects the settings of $\mathrm{pH}$ value generated from the system tracking. Where, predictive length of time domain $^{P}=10$ in Figure 5, controlled length of time domain: $M=1$, weighted coefficient: 0.6. $P=10, M=4$ and weighted coefficients are the same and taken as 0.6, shown in Figure 6. From Figure 5 and Figure 6 it has been seen, the settings of $\mathrm{pH}$ value can be finely tracked down by Genetic algorithm-based NMPC. When $M=1$, the settings of $\mathrm{pH}$ value will be turned into 7 from 10 and then into 13, it lasts for about 2 minutes due to slow response from this system, but existed smaller errors, without system overshoot. When ${ }^{M}=4$, the settings of $\mathrm{pH}$ value will be turned into 7 from 10 and then into 13 , it lasts for about 50 seconds due to fast response from this system, but existed larger errors, with larger system overshoot appeared. Therefore, we can conclude that, when length of controlled time domain is decreased, so does the de-interlacing in the system, but stability and robustness are somewhat increased then. Better control effects can be achieved by reasonable selection of parameters.

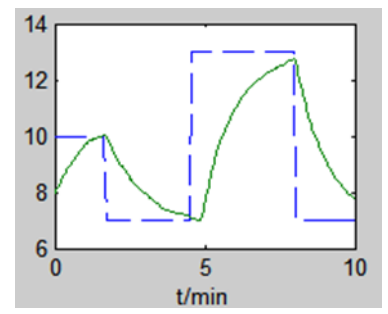

Figure 5. The Tracking Curves when $M=1$

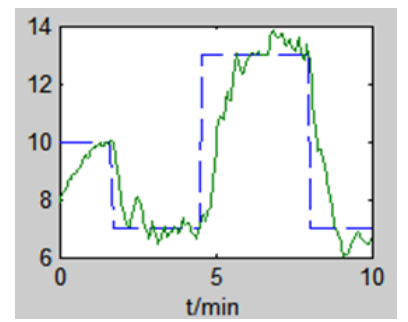

Figure 6. The Tracking Curves when ${ }=4$

\section{Conclusions}

Genetic algorithm-based NMPC will be used in the control system, firstly established a system NMPC model, then the model's moving optimization performed in the system by genetic algorithm, finally, performed the feedback calibration. Genetic algorithm has shortened the NMPC optimized time, Figure ured out the issue of optimization difficulty. By simulation test it is identified that this method can make a better control for $\mathrm{pH}$ neutralization process. Meanwhile, this method also finds its application in the industrial control process with stronger nonlinearity. 


\section{References}

[1] M. Lin, W. G. Lin and D. J. Mo, "Instrumentation pH Value Control System in the Sewage Disposal Based on Fuzzy Adaptation Control PID", Technology and Sensor, (2008).

[2] X. Z. He, Y. J. Guo and L. He, "The Research on Advanced Control for pH Value", In the Process of Zinc Hydrometallurgy in the Instrumentation, Technology and Sensor, (2008).

[3] J. Zhu, "The Control Application for Intelligence Prediction", (2002).

[4] T. Zhu, "Wang Jing-chun:DE-based Nonliner Model Predictive Control of a pH neutralization Process", Acta Automatic Sinica, vol. 36, no.1, (2012).

[5] H. S. Guo and Z. J. Liu, "Design and Application of PLC Fuzzy Controller in Sewage Disposal Process", Programmable Controller \& Factory Automation, (2011).

[6] B. Y. Ji and J. Xiao, "PI-Type Generalized Predictive Control Based on GA Parameter Optimization and Tuning", Science Technology and Engineering, (2011).

[7] S. C. Song and H. Pang, "Research of Text Categorization Based on Genetic Algorithm and Support Vector Machine", Computer Simulation, (2011). 
International Journal of Control and Automation Vol. 8, No. 1 (2015) 